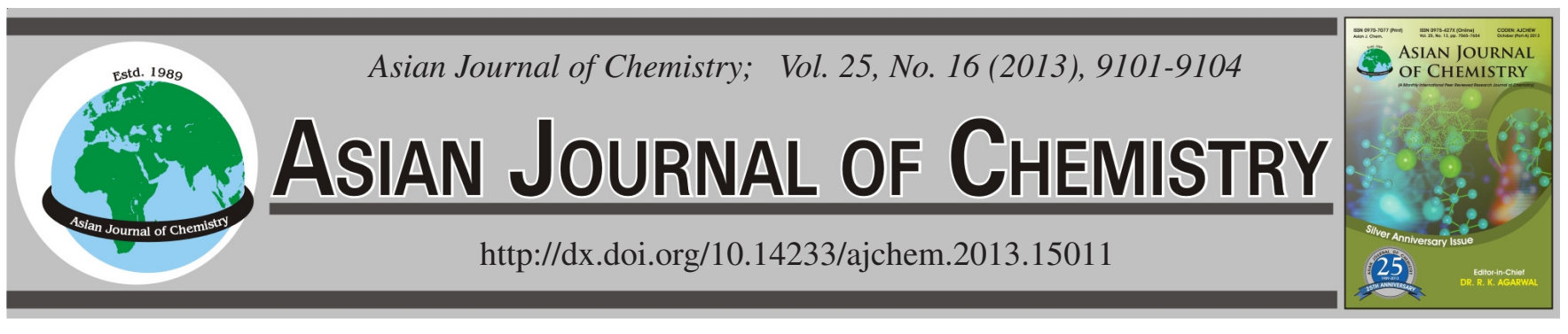

\title{
Preparation, Structure and Biological Activities of Exopolysaccharides Produced by Enterobacter cloacae
}

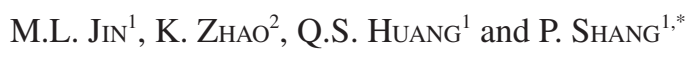

${ }^{1}$ School of Life Sciences, Northwestern Polytechnical University, Xi'an 710072, P.R. China

${ }^{2}$ College of Medicine, Xi'an Jiaotong University, Xi'an 710061, P.R. China

*Corresponding author: Fax: +86 29 88491671; Tel: +86 29 88460391; E-mail: shangpeng@nwpu.edu.cn

\begin{abstract}
Bacterial exopolysaccharides have been proved to have a wide range of health benefits effects. Enterobacter cloacae, a bacterial strain, can produce large amounts of exopolysaccharides. In the last few years, the exopolysaccharides produced by $E$. cloacae have attracted considerable research attention. Many studies on pharmacology have demonstrated that these macromolecules had various biological activities, such as immunomodulation, antioxidant, antitumor and antidiabetes. The aim of the present report is to summarize previous and current references and give a comprehensive summary of information regarding preparation, structural features as well as biological activities of these exopolysaccharides produced by E. cloacae.
\end{abstract}

Key Words: Exopolysaccharide, Enterobacter cloacae, Preparation, Structure, Bioactivities.

\section{INTRODUCTION}

Microorganisms can synthesize a wide spectrum of multifunctional polysaccharides including intracellular polysaccharides, structural polysaccharides and exopolysaccharides. In recent years, a large number of exopolysaccharides have attracted considerable research attention because of their specific functions ${ }^{1}$. Owing to the wide diversity in composition, these macromolecules have found multifarious applications in various pharmaceutical and chemical industries. Bacterial exopolysaccharides have been proved to have a wide range of health benefits effects ${ }^{2}$. They may be developed as one of the useful candidates in the search for effective, non-toxic substances with immunomodulatory, antioxidant, antitumor activity, etc. ${ }^{3}$.

Enterobacter cloacae, a bacterial strain, can produce large amounts of exopolysaccharides. In the last few years, the exopolysaccharides produced by E. cloacae (EPS) have attracted much attention. They have been used as bioflocculant ${ }^{4}$, bioemulsifier ${ }^{5}$ and heavy metal removal agent ${ }^{6}$. Furthermore, many studies on pharmacology have demonstrated that exopolysaccharides produced by E. cloacae had various biological activities, such as immunomodulation ${ }^{7-10}$, antioxidant ${ }^{11,12}$, antitumor ${ }^{13}$ and antidiabetes ${ }^{14}$. Therefore, exopolysaccharides produced by $E$. cloacae have great potential for further development as products in pharmaceutical areas. The aim of the present review is to summarize previous and current references and give a comprehensive summary of information regarding preparation, structural features as well as biological activities of exopolysaccharides in order to provide new insight for further development of these macromolecules.

Fermentation and preparation methods: Exopolysaccharides are high-molecular-weight polymers that are secreted by the microorganism into the surrounding environment. Thus, fermentation is commonly used to prepare exopolysaccharides produced by bacterium. Jin $\mathrm{et} \mathrm{al.}{ }^{8}$ performed exopolysaccharides production in a $10 \mathrm{dm}^{3}$ bioreactor at $30{ }^{\circ} \mathrm{C}$ for 2 days. The cultivation medium contained $2.5 \%$ dextrose, $0.5 \%$ peptone, $0.5 \%$ yeast extract, $0.2 \% \mathrm{~K}_{2} \mathrm{HPO}_{4}$, $0.1 \% \mathrm{KH}_{2} \mathrm{PO}_{4}$ and $0.05 \% \mathrm{MgSO}_{4} \cdot 7 \mathrm{H}_{2} \mathrm{O}$. The inoculation volume was $5 \%(\mathrm{v} / \mathrm{v})$ and the initial $\mathrm{pH}$ was 7.5 with the aeration rate of $1 \mathrm{vvm}$. Prasertsan et al..$^{15}$ optimized the medium and environmental conditions for process development of exopolysaccharides from E. cloacae $\mathrm{WD}^{7}$, they found that the optimum medium contained $3 \%$ sucrose, $0.05 \%$ yeast extract, no addition of any nitrogen source in the basal medium with the initial $\mathrm{pH}$ of 7. The optimum condition during cultivation was controlling $\mathrm{pH}$ at 7 at $30^{\circ} \mathrm{C}$ with the aeration rate of $2.0 \mathrm{vvm}$. In another investigation carried out by Jin et al. ${ }^{1}$, it was found that the concentration of carbon source (maltose) and nitrogen source (a combination of tryptone and beef extract) were the major constituents affecting the yield of exopolysaccharides produced by E. cloacae Z0206. They further optimized the medium composition of above three factors to increase the 
yield of exopolysaccharides using response surface methodology and the optimal medium was determined as $0.05 \%$ $\mathrm{KNO}_{3}, 0.3 \% \mathrm{~K}_{2} \mathrm{HPO}_{4}, 0.15 \% \mathrm{MgSO}_{4}, 3.72 \%$ maltose, $0.51 \%$ tryptone and $0.56 \%$ beef extract. Under the optimal conditions, the yield of exopolysaccharides was $12.95 \mathrm{~g} / \mathrm{L}$ of fermentation liquor, which was verified by validation experiments.

After cultivation, the mycelia could be removed from the broth by centrifugation. The supernatant should be collected and evaporated under reduced pressure, then precipitated upon addition of 3 vol. of $95 \%$ ethanol. The resulting precipitates could be redissolved and deproteinized by a combination of papain and trypsin enzymolysis and the Sevag method ${ }^{16}$. After dialysis or ultrafiltration, ethanol precipitation and drying under vacuum, the crude exopolysaccharides could be obtained. The content of polysaccharide could be determined by the phenolsulphuric method ${ }^{17}$ and the protein could be quantified according to the Bradford method ${ }^{18}$. A combination of techniques, such as fractional precipitation, ion-exchange chromatography, gel filtration and affinity chromatography, could be further used to purify the crude exopolysaccharides. Jin et al. ${ }^{8}$ isolated the purified exopolysaccharides by DEAE-52 and Sephadex G-100 column chromatography, while Wang et al. ${ }^{19}$ purified exopolysaccharides by DEAE-52 and Superose column chromatography.

Structural features: The structural features of a polysaccharide are defined by molecular weight, composition and sequence of monosaccharide, configuration and position of glycosidic linkages, type and polymerization degree of branch and spatial configuration etc. ${ }^{20}$. All of the exopolysaccharides isolated from the submerged culture broth of E. cloacae reported in literatures are heteropolysaccharides. Their structures have been studied using a combination of techniques, such as gel-permeation chromatography, methylation analysis, periodate oxidation, Smith degradation, high performance liquid chromatography (HPLC), gas chromatography-mass spectrometry (GC-MS), electrospray ionization mass spectrometry (ESI-MS), Fourier transform infrared (FTIR) spectra and nuclear magnetic resonance (NMR) spectra. Jin et al. ${ }^{8}$ reported that a water-soluble exopolysaccharide named as EPS1 produced by E. cloacae Z0206 was composed of glucose, mannose and galactose in a molar ratio of 6.860:1.180:0.455 with the average molecular weight of $23928 \mathrm{Da}$ and it hypothesized that EPS- 1 belonged to the $\alpha$-type heteropolysaccharide with pyran group. Wang et al..$^{19}$ recently isolated a novel high molecular weight $\left(1.1 \times 10^{6} \mathrm{Da}\right)$ exopolysaccharides produced by E. cloacae Z0206 by DEAE-52 and Superose column chromatography. Complete hydrolysis of exopolysaccharides followed by GC-MS and HPLC analyses showed that exopolysaccharides was composed of fucose, glucose, galactose, glucuronic acid and pyruvic acid in the approximate molar ratio of $2: 1: 3: 1: 1$. A combination of chemical analysis coupled with ESI-MS as well as NMR analyses indicated that exopolysaccharides comprised a heptasaccharide repeating unit as showed in Fig. 1.

Immunomodulatory activity: The high molecular weight polysaccharides are often called biological response modifiers or immunopotentiators and it is generally accepted that they can enhance various immune responses both in vitro and in

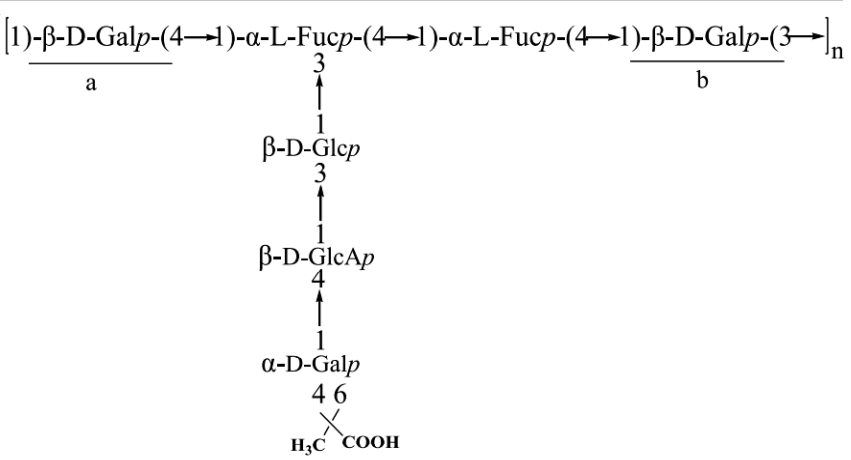

${ }^{\mathrm{ab}}$ may be interchangeable

Fig. 1. Structure of exopolysaccharide produced by E. cloacae Z0206 ${ }^{18}$

vivo ${ }^{21}$. Lu et al. ${ }^{7}$ reported that administration of exopolysaccharides at a dose of $0.4 \%$ in basal diets for 28 days significantly increased the spleen and thymus indices, concentrations of immunoglobulin $\mathrm{G}(\mathrm{IgG})$ and immunoglobulin $\mathrm{M}$ (IgM) and levels of tumor necrosis factor $\alpha(\mathrm{TNF}-\alpha)$, interleukin (IL)-2 and IL-6 in the serum in SD rats. As for immunosuppressed mice induced by cyclophosphamide (CP), oral administration of exopolysaccharides ( $400 \mathrm{mg} / \mathrm{kg}$ body weight) could markedly improve B lymphocyte proliferation and TNF- $\alpha$ production in the serum ${ }^{8}$. IgG and IgM are involved in the complement activation, opsonization and neutralization of toxins $^{22}$. The production of cytokines is a key event in the initiation and regulation of immune response and lymphocyte proliferation plays a crucial role in the activation cascade of both the cellular and humoral immune responses ${ }^{2}$. The above results indicated that exopolysaccharides has potent effect on the cellular and humoral immune responses and it may act as an effective immunomodulatory agent.

Selenium is an essential micronutrient, which is actively involved in animal physiology via a variety of selenoproteins ${ }^{14}$. It has been found that E. cloacae Z0206 could accumulate Se in the form of Se-enriched exopolysaccharide (Se-ECZ-EPS) efficiently during cultivation with selenium ${ }^{2}$. To investigate the immunomodulation effects of Se-ECZ-EPS produced by E. cloacae Z0206, Wang et al. ${ }^{9}$ examined phenoloxidase (PO) activity, respiratory burst (RB) ability, superoxide dismutase (SOD) level and several immune-related genes' expression profile in red swamp crayfish that were individually induced with Se-ECZ-EPS at $1 \mu \mathrm{g} / \mathrm{g}$. It was found that PO, RB and SOD activities of crayfish increased significantly at 24, 48 and $72 \mathrm{~h}$ after being injected with Se-ECZ-EPS. The mRNA expressions of serine proteinase, HSP70 and Mn-SOD were significantly up-regulated at 24 and $48 \mathrm{~h}$ after Se-ECZ-EPS treatment. Meanwhile, Se-ECZ-EPS treatment could obviously improve the disease resistance of crayfish against white spot syndrome virus. These results indicated that Se-ECZ-EPS is an efficient immunostimulant and it can improve the immunity of crayfish.

Antioxidant activity: Oxidative stress is a crucial mediator of cell injury and pathology. Reactive oxygen species (ROS) and free radicals can lead to the induction of membrane damage and the oxidation of biomacromolecules such as DNA, cellular lipids and proteins ${ }^{23}$. Therefore, the development of novel antioxidants to prevent oxidative damage in the food and 
pharmaceutical industry has attracted more and more attention over the past few years. The antioxidant activities of exopolysaccharides have been studied both in vitro and in vivo previously. It was found that exopolysaccharides possessed considerable scavenging activities against 1,1-diphenyl-2picrylhydrazyl (DPPH) radical and hydroxyl radical in a concentration-dependent manner ${ }^{11,12}$. At the concentration of $5 \mathrm{mg} / \mathrm{mL}$, the scavenging effect of exopolysaccharides on DPPH radical and hydroxyl radical reached to 61.57 and 40.48 $\%$, respectively. Jin et al. ${ }^{8}$ investigated the antioxidant activity of exopolysaccharides against $\mathrm{CP}$-induced oxidative damage in mice. They found that oral administration of exopolysaccharides to CP-exposed animals ( $400 \mathrm{mg} / \mathrm{kg}$ body weight) resulted in a significant increase of activities of antioxidant enzymes such as SOD and glutathione peroxidase (GPx) in hepatocytes. It suggested that exopolysaccharides treatment might ameliorate the synthesis of essential antioxidant enzymes, which have a role in preventing the pathological concentrations of ROS.

The biological activities of polysaccharide mainly depend on its molecular structures. Many investigations have demonstrated that sulfated modification of some natural polysaccharides could not only enhance the water solubility but also change the chain conformation, resulting in the improvement of their biological activities ${ }^{24,25}$. Therefore, sulfated modification may be used to enhance the bioactivities of some natural polysaccharides. Jin et al. ${ }^{26}$ prepared the sulfated derivatives of exopolysaccharides with different degrees of substitution (DS) by chlorosulfonic acid-pyridine method. Their antioxidant activities, such as scavenging abilities on superoxide radical and hydroxyl radical, were evaluated in vitro. It was found the sulfated derivatives of exopolysaccharides showed noticeable effects on scavenging superoxide radical and hydroxyl radical compared with native one and sulfated derivative with moderate degrees of substitution of 0.60 showed highest antioxidant activities. The scavenging effect of sulfated derivative with degrees of substitution of 0.60 on superoxide radical was $89.06 \%$ at the concentration of $2.0 \mathrm{mg} / \mathrm{mL}$, while at the same concentration, the scavenging effect of vitamin $\mathrm{C}$ was only $80.66 \%$. These results suggested sulfate modification could be considered as the effective approach to enhance the antioxidant activities of exopolysaccharides.

Antitumor activity: Zhang et $a l .{ }^{13}$ previously isolated and fractionated exopolysaccharides from E. cloacae using a combination of techniques, such as ultrasonic, cetyltrimethylammonium bromide and ethanol precipitation and DEAE cellulose column chromatography. It was found that F3, one of the glycoprotein components, could distinctly inhibit the growth of QGY7703 (liver cancer), A549 (glandular cancer of the lungs), KatoIII (gastric carcinoma) and SW1116 (intestinal cancer) cell lines in vitro. Its $50 \%$ inhibition concentration $\left(\mathrm{IC}_{50}\right)$ to these tumor cells were $0.024,0.0123,0.0035$ and $0.012 \mathrm{mg} / \mathrm{mL}$, respectively. Furthermore, F3 could inhibit the proliferation of Lewis lung cancer in mice with a dose-dependent manner.

Antidiabetic activity: Diabetes mellitus is a serious chronic metabolic disease which now afflicts $4 \%$ of population worldwide and is expected to increase by $5.4 \%$ in $2025^{27}$.
Hence, over the past few years there has been an increased interest in the exploration of novel naturally occurring agents with anti-diabetic activities to prevent the development of diabetes. Jin et al. ${ }^{14}$ isolated the water-soluble Se-ECZ-EPS from submerged culture broth of E. cloacae Z0206. The protective effects of Se-ECZ-EPS on alloxan-induced diabetic mice were investigated. Diabetes was induced in ICR (Institute of Cancer Research) mice by administration of single doses of alloxan intraperitoneally (190 mg/kg body weight). The decrease in body weight, serum insulin level and the increase in blood glucose level, glycosylated serum protein (GSP), total cholesterol (TC) and triglycerides (TG) in the liver were observed in diabetic mice. While oral administration of Se-ECZ-EPS (200 mg/kg body weight) could significantly improve the body weight and serum insulin level and obviously decrease the fasting blood glucose levels, glycosylated serum protein, total cholesterol and triglycerides contents in the liver of diabetic mice. It suggested that Se-ECZ-EPS possesses significant antidiabetic effects in alloxan-induced diabetic mice and it may have a potential clinical utility in treating diabetic patients.

\section{Conclusion}

In the last few years, the exopolysaccharides produced by E. cloacae have been developed to be used as bioflocculants, bioemulsifier and heavy metal removal agents. Furthermore, many studies on pharmacology have demonstrated that these exopolysaccharides had various biological activities, such as immunomodulation, antioxidant, antitumor and antidiabetes. However, most of these bioactivities were investigated in vitro or in animal model. Further studies of the bioactive effects on human subjects are required. Meanwhile, the relationship between bioactivities and chemical structures of these macromolecules are needed to be established, which would provide new insight for further development of natural resources.

\section{ACKNOWLEDGEMENTS}

This study was supported by the International Foundation for Science (Grant No. F/5206-1) and China Postdoctoral Science Foundation (Grant Nos. 2011M501481 and 2012T50821).

\section{REFERENCES}

1. M.L. Jin, Y.M. Wang, M. Huang, Z.Q. Lu and Y.Z. Wang, Asian J. Chem., 23, 3799 (2011).

2. C.L. Xu, Y.Z. Wang, M.L. Jin and X.Q. Yang, Bioresour. Technol., 100, 2095 (2009).

3. H.Y. Wang, X.L. Jiang, H.J. Mu, X.T. Liang and H.S. Guan, Microbiol. Res., 162, 124 (2007).

4. P. Prasertsan, W. Dermlim, H. Doelle and J.F. Kennedy, Carbohydr. Polym., 66, 289 (2006).

5. X. Hua, Z. Wu, H. Zhang, D. Lu, M. Wang, Y. Liu and Z. Liu, Chemosphere, 80, 951 (2010).

6. M.M. Naik, A. Pandey and S.K. Dubey, Biodegradation, 23, 775 (2012).

7. Z. Lu, X. Yang, M. Jin, Y. Cao and Y. Wang, Chin. J. Anim. Nutr., 22, 1350 (2010).

8. M. Jin, Y. Wang, C. Xu, Z. Lu, M. Huang and Y. Wang, Carbohydr. Polym., 81, 607 (2010).

9. X. Wang, Z. Lu, L. Zhu, Y. Zhang, Y. Ren and Y. Wang, Aquac. Res., 41, e819 (2010).

10. X.Q. Yang, M.L. Jin, C.L. Xu, T.Z. Shan and Y.Z. Wang, J. Agric. Biotechnol., 17, 815 (2009).

11. C.L. Xu, C.G. Qin, W.N. Niu and X.Y. Shang, Nat. Prod. Res. Dev., 22, 1098 (2010). 
12. C.L. Xu, C.G. Qin, X.Y. Shang and W.N. Niu, Chem. Res., 21, 64 (2010).

13. M.J. Zhang, Q.G. Ren and Y.Q. Chen, J. Fudan Univ. Nat. Sci., 41, 378 (2002).

14. M. Jin, Z. Lu, M. Huang, Y. Wang and Y. Wang, Int. J. Biol. Macromol., 50, 348 (2012).

15. P. Prasertsan, S. Wichienchot, H. Doelle and J.F. Kennedy, Carbohydr Polym., 71, 468 (2008)

16. A.M. Staub, Method Carbohydr. Chem., 5, 5 (1965).

17. M. Dubois, K.A. Gilles, J.K. Harmilton, P.A. Rebers and F. Smith, Anal. Chem., 28, 350 (1956).

18. M.M. Bradford, Anal. Biochem., 72, 248 (1976).

19. F. Wang, H. Yang and Y. Wang, Carbohydr. Polym., 92, 503 (2013).

20. S.P. Nie and M.Y. Xie, Food Hydrocolloids, 25, 144 (2011)

21. C. Liu, Q. Lin, Y. Gao, L. Ye, Y. Xing and T. Xi, Carbohydr. Polym., 67, 313 (2007)
22. L. Guo, J. Liu, Y. Hu, D. Wang, Z. Li, J. Zhang, T. Qin, X. Liu, C. Liu, X. Zhao, Y.P. Fan, G. Han and T.L. Nguyen, Carbohydr. Polym., 90, 1055 (2012).

23. M. Jin, K. Zhao, Q. Huang, C. Xu and P. Shang, Carbohydr. Polym., 89, 713 (2012).

24. X. Huang, Y. Hu, X. Zhao, Y. Lu, J. Wang, F. Zhang and J. Sun, Carbohydr. Polym., 73, 303 (2008).

25. S. Chen, J. Wang, C. Xue, H. Li, B. Sun, Y. Xue and W. Chai, Carbohydr. Polym., 81, 560 (2010).

26. M. Jin, Z. Lu, M. Huang, Y. Wang and Y. Wang, Int. J. Biol. Macromol., 48, 607 (2011).

27. I. Dahech, K.S. Belghith, K. Hamden, A. Feki, H. Belghith and H. Mejdoub, Int. J. Biol. Macromol., 49, 742 (2011). 